\title{
Modulation space estimates for multilinear pseudodifferential operators
}

by

ÁRPÁD BÉNYi (Bellingham, WA) and KAsso A. OKoudjou (Ithaca, NY)

Abstract. We prove that for symbols in the modulation spaces $\mathcal{M}^{p, q}, p \geq q$, the associated multilinear pseudodifferential operators are bounded on products of appropriate modulation spaces. In particular, the symbols we study here are defined without any reference to smoothness, but rather in terms of their time-frequency behavior.

1. Introduction. An $m$-linear pseudodifferential operator is a priori defined through its (distributional) symbol $\sigma$ as a mapping from the $m$-fold product $\mathcal{S}\left(\mathbb{R}^{d}\right) \times \cdots \times \mathcal{S}\left(\mathbb{R}^{d}\right)$ into $\mathcal{S}^{\prime}\left(\mathbb{R}^{d}\right)$ by

$$
\begin{aligned}
& T_{\sigma}\left(f_{1}, \ldots, f_{m}\right)(x) \\
& \quad=\int_{\mathbb{R}^{m d}} \sigma\left(x, \xi_{1}, \ldots, \xi_{m}\right) \widehat{f}_{1}\left(\xi_{1}\right) \cdots \widehat{f}_{m}\left(\xi_{m}\right) e^{2 \pi i x \cdot\left(\xi_{1}+\cdots+\xi_{m}\right)} d \xi_{1} \cdots d \xi_{m}
\end{aligned}
$$

for $f_{1}, \ldots, f_{m} \in \mathcal{S}\left(\mathbb{R}^{d}\right)$. Such operators model the product of $m$ functions and their derivatives and one expects their boundedness properties on products of Lebesgue spaces to mimic those given by Hölder's inequality. The main question then is to find sufficient optimal conditions on the symbol $\sigma$ that guarantee the boundedness of the corresponding operator $T_{\sigma}$ on various spaces of functions. The search for such conditions can be traced back to the pioneering work of Coifman and Meyer [9-11]. They have proved, for instance, that (if we assume for simplicity $m=2$ ) the conditions

$$
\left|\partial_{\xi}^{\beta} \partial_{\eta}^{\gamma} \sigma(x, \xi, \eta)\right| \leq C_{\beta, \gamma}
$$

and

$$
\left|\partial_{\xi}^{\beta} \partial_{\eta}^{\gamma} \sigma\left(x^{\prime}, \xi, \eta\right)-\partial_{\xi}^{\beta} \partial_{\eta}^{\gamma} \sigma(x, \xi, \eta)\right| \leq C_{\beta, \gamma}\left|x^{\prime}-x\right|^{\delta}
$$

for all $\beta, \gamma \geq 0$ and some $\delta>0$ imply that $T_{\sigma}$ is bounded from $L^{p} \times L^{q}$ into $L^{r}$ when $1 / p+1 / q=1 / r, p, q>1$.

2000 Mathematics Subject Classification: Primary 47G30; Secondary 42B35, 35S99.

Key words and phrases: modulation spaces, multilinear operators, pseudodifferential operators, short-time Fourier transform. 
The ideas of Coifman and Meyer have had far reaching consequences and have led, in particular, to the investigation of bilinear and multilinear pseudodifferential operators in the realm of other classical function spaces, such as Sobolev or Besov-Triebel-Lizorkin spaces in the works of Grafakos and Torres [19, 20]; see also [1, 3, 5-7]. In all these papers the recurrent assumptions on the symbol are sufficient smoothness and decay conditions, as well as size estimates.

More recently, in [4], we have initiated the study of multilinear pseudodifferential operators in the context of modulation spaces, which surprisingly appear as the right spaces in certain problems where more common $L^{p}$ estimates fail. These spaces play a dual role of classes of symbols and spaces of functions on which the operators act. For example, we proved in [4] that symbols in the so-called Feichtinger algebra yield bilinear operators that are bounded on products of modulation spaces. As a corollary, we also obtained boundedness results for operators in this class on products of Lebesgue and Sobolev spaces. It is important to note that the Feichtinger algebra as well as all the family of modulation spaces it belongs to are defined without any reference to smoothness, but rather by imposing some decay conditions on the time-frequency content of functions; see Gröchenig's book [15] for further details about modulation spaces. The analysis of multilinear pseudodifferential operators in the realm of modulation spaces was continued in [2], where it was shown that the modulation space $\mathcal{M}^{\infty, 1}\left(\mathbb{R}^{(m+1) d}\right)$ also yields bounded operators on products of modulation spaces. The space $\mathcal{M}^{\infty, 1}$ has played an important role in the recent development of the theory of linear pseudodifferential operators; see the works of Gröchenig and Heil [16-18], Heil, Ramanathan, and Topiwala [21], Labate [22, 23], Sjöstrand [25], and Toft [26]. The results in [2] could be viewed as multilinear extension of the celebrated Calderón-Vaillancourt theorem [8] about the $L^{2}$ boundedness of linear operators with bounded symbols having all their derivatives bounded. Indeed, it can be shown that symbols satisfying such conditions also belong to the modulation space $\mathcal{M}^{\infty, 1}$; see $[24,16,21]$. We also wish to point out that the conditions on the symbols we employed in [4] and [2] are not comparable to the ones previously used in the "hard analysis" works cited above.

In this paper, we investigate the boundedness properties of multilinear pseudodifferential operators with symbols in more general modulation spaces. More precisely, we show that symbols in modulation spaces $\mathcal{M}^{p, q}\left(\mathbb{R}^{(m+1) d}\right), p \geq q$, give rise to bounded operators on products of corresponding modulation spaces; see the article of Czaja [12] and [17, 18] for analogous results in the linear case. As a by-product of our analysis, we improve one of the main results in [4]. More precisely, we prove that if the symbol of a bilinear (or multilinear) pseudodifferential operator 
is in the Feichtinger algebra $\mathcal{M}^{1}$, then the corresponding operator maps $\mathcal{M}^{\infty} \times \mathcal{M}^{\infty}$ into $\mathcal{M}^{1}$, and hence it maps $\mathcal{M}^{p_{1}, q_{1}} \times \mathcal{M}^{p_{2}, q_{2}}$ into $\mathcal{M}^{1}$ for all indices $1 \leq p_{i}, q_{i} \leq \infty$.

Our paper is organized as follows. In Section 2 we set the notation and the fundamental facts about the modulation spaces that will be used throughout the paper. In Section 3 we state our main results. Their proofs and some corollaries are presented in Section 4.

\section{Notation and preliminaries}

2.1. General notation. We will be working on the $d$-dimensional space $\mathbb{R}^{d}$. We let $\mathcal{S}=\mathcal{S}\left(\mathbb{R}^{d}\right)$ be the subspace of $C^{\infty}\left(\mathbb{R}^{d}\right)$ of Schwartz rapidly decreasing functions, with its usual topology. Its dual is $\mathcal{S}^{\prime}=\mathcal{S}^{\prime}\left(\mathbb{R}^{d}\right)$, the set of all tempered distributions on $\mathbb{R}^{d}$. Translation and modulation of a function $f$ with domain $\mathbb{R}^{d}$ are defined, respectively, by

$$
T_{x} f(t)=f(t-x) \quad \text { and } \quad M_{y} f(t)=e^{2 \pi i y \cdot t} f(t) .
$$

The Fourier transform of $f \in L^{1}\left(\mathbb{R}^{d}\right)$ is $\widehat{f}(\omega)=\int_{\mathbb{R}^{d}} f(t) e^{-2 \pi i t \cdot \omega} d t, \omega \in \mathbb{R}^{d}$. The Fourier transform is an isomorphism of the Schwartz space $\mathcal{S}$ onto itself, and extends to the space $\mathcal{S}^{\prime}$ of tempered distributions by duality.

The inner product of two functions $f, g \in L^{2}$ is $\langle f, g\rangle=\int_{\mathbb{R}^{d}} f(t) \overline{g(t)} d t$, and its extension to $\mathcal{S}^{\prime} \times \mathcal{S}$ will also be denoted by $\langle\cdot, \cdot\rangle$.

The Short-Time Fourier Transform (STFT) of a function $f$ with respect to a window $g$ is

$$
V_{g} f(x, y)=\left\langle f, M_{y} T_{x} g\right\rangle=\int_{\mathbb{R}^{d}} e^{-2 \pi i y \cdot t} \overline{g(t-x)} f(t) d t,
$$

whenever the integral makes sense. Similar to the Fourier transform, the STFT extends in a distributional sense to $f, g \in \mathcal{S}^{\prime}$; see Folland's book [14, Prop. 1.42].

An important technical tool used throughout this paper is the extended isometry property of the STFT [15, (14.31)]: if $\phi \in \mathcal{S}\left(\mathbb{R}^{d}\right),\|\phi\|_{2}=1$, then

$$
\langle f, h\rangle=\left\langle V_{\phi} f, V_{\phi} h\right\rangle, \quad \forall f \in \mathcal{S}^{\prime}, h \in \mathcal{S} .
$$

We let $L^{q} L^{p}=L^{q} L^{p}\left(\mathbb{R}^{2 d}\right)$ be the space of measurable functions $f(x, y)$ for which the mixed norm

$$
\|f\|_{L^{q}(d y) L^{p}(d x)}=\left(\int_{\mathbb{R}^{d}}\left(\int_{\mathbb{R}^{d}}|f(x, y)|^{p} d x\right)^{q / p} d y\right)^{1 / q}
$$

is finite. If $p=q$, we have $L^{p} L^{p}\left(\mathbb{R}^{2 d}\right)=L^{p}\left(\mathbb{R}^{2 d}\right)$, the usual Lebesgue space.

2.2. Modulation spaces. Given $1 \leq p, q \leq \infty$, and given a fixed, non-zero window function $g \in \mathcal{S}$, the modulation space $\mathcal{M}^{p, q}=\mathcal{M}^{p, q}\left(\mathbb{R}^{d}\right)$ is the space 
of all distributions $f \in \mathcal{S}^{\prime}$ for which the following norm is finite:

$$
\|f\|_{\mathcal{M}^{p, q}}=\left(\int_{\mathbb{R}^{d}}\left(\int_{\mathbb{R}^{d}}\left|V_{g} f(x, y)\right|^{p} d x\right)^{q / p} d y\right)^{1 / q}=\left\|V_{g} f\right\|_{L^{q} L^{p}},
$$

with the usual modifications if $p$ and/or $q$ are infinite. When $p=q$, we write $\mathcal{M}^{p}$ for $\mathcal{M}^{p, p}$. Note that for $p=q=2, \mathcal{M}^{2}=L^{2}$.

REMARK 1 . When $p=q=1$ the resulting modulation space $\mathcal{M}^{1}$ is also known as the Feichtinger algebra. It is sometimes denoted $\mathcal{S}_{0}$, and it has some remarkable properties; see [13] for a detailed description. In [4], we proved that $\mathcal{M}^{1}$ as well as some of its weighted versions are convenient classes of symbols that give rise to bounded bilinear pseudodifferential operators on products of modulation spaces.

REMARK 2. The definition of modulation spaces is independent of the choice of the window $g$ in the sense of equivalent norms. It is important to note that the Schwartz class $\mathcal{S}$ is dense in $\mathcal{M}^{p, q}$ for $1 \leq p, q<\infty$. One can also show that the dual of $\mathcal{M}^{p, q}$ is $\mathcal{M}^{p^{\prime}, q^{\prime}}$, where $1 \leq p, q<\infty$ and $1 / p+1 / p^{\prime}=1 / q+1 / q^{\prime}=1$.

REMARK 3. Most of our results will be stated even for the cases $p=\infty$ and/or $q=\infty$. To properly deal with duality (which will be used constantly in our proofs) in these cases, we define the related modulation spaces $\mathcal{M}^{0, q}, \mathcal{M}^{p, 0}$, and $\mathcal{M}^{0,0}=\mathcal{M}^{0}$. Let $L^{0}$ denote the space of bounded measurable functions on $\mathbb{R}^{2 d}$ vanishing at infinity. Then

$$
\begin{array}{ll}
\mathcal{M}^{0, q}=\left\{f \in \mathcal{M}^{\infty, q}: V_{g} f \in L^{0}\right\}, & q<\infty, \\
\mathcal{M}^{p, 0}=\left\{f \in \mathcal{M}^{p, \infty}: V_{g} f \in L^{0}\right\}, & p<\infty, \\
\mathcal{M}^{0,0}=\left\{f \in \mathcal{M}^{\infty}: V_{g} f \in L^{0}\right\}, &
\end{array}
$$

equipped with the norms of $\mathcal{M}^{\infty, q}, \mathcal{M}^{p, \infty}$, and $\mathcal{M}^{\infty, \infty}=\mathcal{M}^{\infty}$ respectively. Moreover, it follows from [2, Lemma 2.2] that $\left(\mathcal{M}^{p, 0}\right)^{\prime}=\mathcal{M}^{p^{\prime}, 1},\left(\mathcal{M}^{0, q}\right)^{\prime}=$ $\mathcal{M}^{1, q^{\prime}}$, and $\left(\mathcal{M}^{0,0}\right)^{\prime}=\mathcal{M}^{1,1}$. From now on we will will use these duality relations in the cases $p=\infty$ and/or $q=\infty$ without any further explanations.

3. Main estimates. Our first main result, of which [2, Theorem 3.1] should be regarded as the limiting case, can be stated as follows.

Theorem 1. Let $\sigma \in \mathcal{M}^{p, 1}\left(\mathbb{R}^{(m+1) d}\right)$ and $1 \leq p, p_{i}, q_{i} \leq \infty, 0 \leq i \leq m$, be such that

$$
\frac{1}{p}+\sum_{i=1}^{m} \frac{1}{p_{i}}=\frac{1}{p_{0}} \quad \text { and } \quad \frac{m}{p}+\sum_{i=1}^{m} \frac{1}{q_{i}}=(m-1)+\frac{1}{q_{0}} .
$$

Then the m-linear pseudodifferential operator $T_{\sigma}$ defined by (1) can be extended to a bounded operator from $\mathcal{M}^{p_{1}, q_{1}} \times \cdots \times \mathcal{M}^{p_{m}, q_{m}}$ into $\mathcal{M}^{p_{0}, q_{0}}$. 
More generally, if we assume that the symbol belongs to $\mathcal{M}^{p, q}\left(\mathbb{R}^{(m+1) d}\right)$, $p \geq q$, then we can prove the following.

Theorem 2. Let $1 \leq p_{0}, p, q, r, q_{i} \leq \infty$ for $0 \leq i \leq m$ be such that

$$
\left\{\begin{array}{l}
p_{0} \leq q_{0} \\
q_{m} \leq r \\
p^{\prime} \leq q^{\prime}, q_{0}^{\prime}, r, q_{i} \quad \text { if } 1 \leq i \leq m, \\
q_{i} \leq q^{\prime} \quad \text { if } 1 \leq i \leq m-1
\end{array}\right.
$$

Moreover, assume that

$$
\frac{m-1}{p}+\frac{1}{q}+\sum_{i=1}^{m} \frac{1}{q_{i}}=m-1+\frac{1}{q_{0}} \quad \text { and } \quad \frac{1}{p}+\frac{1}{q}+\frac{1}{r}=1+\frac{1}{p_{0}} .
$$

If $\sigma \in \mathcal{M}^{p, q}\left(\mathbb{R}^{(m+1) d}\right)$, then the $m$-linear pseudodifferential operator $T_{\sigma}$ defined by (1) can be extended to a bounded operator from $\mathcal{M}^{q^{\prime}, q_{1}} \times \mathcal{M}^{q^{\prime}, q_{2}} \times$ $\cdots \times \mathcal{M}^{q^{\prime}, q_{m-1}} \times \mathcal{M}^{r, q_{m}}$ into $\mathcal{M}^{p_{0}, q_{0}}$.

4. Proofs. It is readily seen that the action of $T_{\sigma}$ on $f_{1}, \ldots, f_{m}, g \in \mathcal{S}$ can be written as

$$
\begin{aligned}
& \left\langle T_{\sigma}\left(f_{1}, \ldots, f_{m}\right), g\right\rangle \\
& =\int_{\mathbb{R}^{(m+1) d}} \sigma\left(x, \xi_{1}, \ldots, \xi_{m}\right) \widehat{f}_{1}\left(\xi_{1}\right) \cdots \widehat{f}_{m}\left(\xi_{m}\right) e^{2 \pi i x \cdot\left(\xi_{1}+\cdots+\xi_{m}\right)} \bar{g}(x) d \xi_{1} \cdots d \xi_{m} d x \\
& =\left\langle\sigma, W_{m}\left(g, f_{1}, \ldots, f_{m}\right)\right\rangle
\end{aligned}
$$

where

$$
\begin{aligned}
W_{m}\left(g, f_{1}, \ldots, f_{m}\right)\left(x, \xi_{1}, \ldots, \xi_{m}\right) & \\
& =g(x) \overline{\widehat{f}_{1}}\left(\xi_{1}\right) \cdots \overline{\widehat{f}_{m}}\left(\xi_{m}\right) e^{-2 \pi i x \cdot\left(\xi_{1}+\cdots+\xi_{m}\right)} .
\end{aligned}
$$

REMARK 4. For $m=1$, the Kohn-Nirenberg correspondence can be written as $\left\langle T_{\sigma} f, g\right\rangle=\left\langle\sigma, W_{1}(g, f)\right\rangle$, where $W_{1}(g, f)=e^{-2 \pi i x \cdot \xi} g(x) \widehat{f}(\xi)$ is the so-called cross Ryhaczek distribution of $f$ and $g$. One may think of $W_{m}$ as a multilinear version of the Ryhaczek distribution. The key idea in obtaining any type of modulation spaces estimates on multilinear pseudodifferential operators $T_{\sigma}$ is to understand the time-frequency characteristics of $W_{m}\left(g, f_{1}, \ldots, f_{m}\right)$.

To compactify the notation, we write

$$
\left(\xi_{1}, \ldots, \xi_{m}\right)=\vec{\xi}, \quad d \xi_{1} \cdots d \xi_{m}=d \vec{\xi}, \quad W_{m}\left(g, f_{1}, \ldots, f_{m}\right)=W_{m}(g, \vec{f}) .
$$

Let now $\left(\phi_{0}, \vec{\phi}\right)=\left(\phi_{0}, \phi_{1}, \ldots, \phi_{m}\right) \in \mathcal{S}\left(\mathbb{R}^{(m+1) d}\right)$, and let $\left(u_{0}, \vec{u}\right)=$ $\left(u_{0}, u_{1}, \ldots, u_{m}\right),\left(v_{0}, \vec{v}\right)=\left(v_{0}, v_{1}, \ldots, v_{m}\right) \in\left(\mathbb{R}^{d}\right)^{m+1}$. Then we clearly have $W_{m}\left(\phi_{0}, \vec{\phi}\right) \in \mathcal{S}\left(\mathbb{R}^{(m+1) d}\right)$. 
The proofs of our main results will be based on the lemma and propositions below. The following result was proved in [2].

Lemma 1. Let $(\vec{f}, g) \in\left(\mathcal{M}^{\infty}\left(\mathbb{R}^{d}\right)\right)^{m+1}$. With the notation above, we have

$$
\begin{aligned}
V_{W_{m}\left(\phi_{0}, \vec{\phi}\right)} & W_{m}(g, \vec{f})\left(\left(u_{0}, \vec{u}\right),\left(v_{0}, \vec{v}\right)\right) \\
= & e^{2 \pi i u_{0} \cdot \sum_{i=1}^{n} u_{i}} V_{\phi_{0}} g\left(u_{0}, v_{0}+\sum_{i=1}^{n} u_{i}\right) \overline{\prod_{i=1}^{m} V_{\phi_{i}} f_{i}\left(u_{0}+v_{i}, u_{i}\right) .}
\end{aligned}
$$

The next proposition contains the main estimate needed for proving Theorem 1.

Proposition 1. Assume that

$$
\frac{1}{p}+\sum_{i=1}^{m} \frac{1}{p_{i}}=\frac{1}{p_{0}} \quad \text { and } \quad \frac{m}{p}+\sum_{i=1}^{m} \frac{1}{q_{i}}=m-1+\frac{1}{q_{0}},
$$

where $1 \leq p, p_{i}, q_{i} \leq \infty, 0 \leq i \leq m$. Then

$$
\begin{aligned}
\left\|V_{W_{m}\left(\phi_{0}, \vec{\phi}\right)} W_{m}(g, \vec{f})\right\|_{L^{\infty}\left(d v_{0} d \vec{v}\right) L^{p^{\prime}}\left(d u_{0} d \vec{u}\right)} & \\
& \leq C\left\|f_{1}\right\|_{\mathcal{M}^{p_{1}, q_{1}}} \cdots\left\|f_{m}\right\|_{\mathcal{M}^{p_{m}, q_{m}}}\|g\|_{\mathcal{M}^{p_{0}^{\prime}, q_{0}^{\prime}}},
\end{aligned}
$$

whenever the right hand side is defined. Equivalently, $W_{m}(g, \vec{f}) \in \mathcal{M}^{p^{\prime}, \infty}$.

Proof. Lemma 1 implies that for all $\left(v_{0}, \vec{v}\right) \in \mathbb{R}^{(m+1) d}$ we have

$$
\begin{aligned}
\Gamma\left(v_{0}, \vec{v}\right)^{p^{\prime}} & =\int_{\mathbb{R}^{(m+1) d}}\left|V_{W_{m}\left(\phi_{0}, \vec{\phi}\right)} W_{m}(g, \vec{f})\left(\left(u_{0}, \vec{u}\right),\left(v_{0}, \vec{v}\right)\right)\right|^{p^{\prime}} d u_{0} d \vec{u} \\
& =\int_{\mathbb{R}^{(m+1) d}}\left|V_{\phi_{0}} g\left(u_{0}, v_{0}+\sum_{i=1}^{n} u_{i}\right)\right|^{p^{\prime}}\left|\prod_{i=1}^{m} V_{\phi_{i}} f_{i}\left(u_{0}+v_{i}, u_{i}\right)\right|^{p^{\prime}} d u_{0} d \vec{u} .
\end{aligned}
$$

Observe that the condition on the exponents $p_{i}$ is equivalent to $1 / p^{\prime}=$ $1 / p_{0}^{\prime}+\sum_{i=1}^{m} 1 / p_{i}$. Hence, if we apply Hölder's inequality we get

$$
\Gamma\left(v_{0}, \vec{v}\right)^{p^{\prime}} \leq \int_{\mathbb{R}^{m d}}\left\|V_{\phi_{0}} g\left(\cdot, v_{0}+\sum_{i=1}^{n} u_{i}\right)\right\|_{L^{p_{0}^{\prime}}}^{p^{\prime}} \prod_{i=1}^{m}\left\|V_{\phi_{i}} f_{i}\left(\cdot, u_{i}\right)\right\|_{L^{p_{i}}}^{p^{\prime}} d \vec{u} .
$$

Let $G(v)=\left\|V_{\phi_{0}} g(\cdot, v)\right\|_{L^{p_{0}^{\prime}}}$ and $F_{i}\left(u_{i}\right)=\left\|V_{\phi_{i}} f_{i}\left(\cdot,-u_{i}\right)\right\|_{L^{p_{i}}}$. With this notation, $\|G\|_{L^{q_{0}^{\prime}}}=\|g\|_{\mathcal{M}^{p_{0}^{\prime}, q_{0}^{\prime}}}$ and $\left\|F_{i}\right\|_{L^{q_{i}}}=\left\|f_{i}\right\|_{\mathcal{M}^{p_{i}, q_{i}}}$. Therefore, we may rewrite the previous inequality as

$$
\begin{aligned}
\Gamma\left(v_{0}, \vec{v}\right)^{p^{\prime}} & \leq \int_{\mathbb{R}^{m d}} G\left(v_{0}+\sum_{i=1}^{n} u_{i}\right)^{p^{\prime}} \prod_{i=1}^{m} F_{i}\left(-u_{i}\right)^{p^{\prime}} d \vec{u} \\
& =\left(G^{p^{\prime}} * F_{1}^{p^{\prime}} * \cdots * F_{m}^{p^{\prime}}\right)\left(v_{0}\right) .
\end{aligned}
$$


Note that the expression on the right hand side of this inequality is already independent of $\vec{v}$. Thus, the only meaningful estimate we may hope for is an $L^{\infty}$ estimate. The condition on the exponents $q_{i}$ is equivalent to $m / p^{\prime}+$ $1 / \infty=1 / q_{0}^{\prime}+\sum_{i=1}^{m} 1 / q_{i}$, and this allows us to use Young's inequality for convolution. We obtain

$$
\begin{aligned}
\left\|V_{W_{m}\left(\phi_{0}, \vec{\phi}\right)} W_{m}(g, \vec{f})\right\|_{L^{p^{\prime}, \infty}}^{p^{\prime}} & \leq\left\|G^{p^{\prime}} * F_{1}^{p^{\prime}} * \cdots * F_{m}^{p^{\prime}}\right\|_{L^{\infty}} \\
& \leq\left\|G^{p^{\prime}}\right\|_{L^{q_{0}^{\prime} / p^{\prime}}} \prod_{i=1}^{m}\left\|F_{i}^{p^{\prime}}\right\|_{L^{q_{i} / p^{\prime}}} \\
& =\|G\|_{L^{q^{\prime}}}^{p^{\prime}} \prod_{i=1}^{m}\left\|F_{i}\right\|_{L^{q_{i}}}^{p^{\prime}} .
\end{aligned}
$$

This proves that

$$
\left\|V_{W_{m}\left(\phi_{0}, \vec{\phi}\right)} W_{m}(g, \vec{f})\right\|_{L^{p^{\prime}, \infty}} \leq C\|g\|_{\mathcal{M}^{p_{0}^{\prime}, q_{0}^{\prime}}}\left\|f_{1}\right\|_{\mathcal{M}^{p_{1}, q_{1}}} \cdots\left\|f_{m}\right\|_{\mathcal{M}^{p_{m}, q_{m}}} .
$$

The appearance of the constant $C$ is due to the use of different windows to measure the modulation space norms and as such it depends only on the dimension and the indices of these spaces.

We come now to the proof of Theorem 1.

Proof of Theorem 1. Let $f_{i} \in \mathcal{M}^{p_{i}, q_{i}}$ be given, and let $\phi_{0}, \phi_{1}, \ldots, \phi_{m} \in$ $\mathcal{S}\left(\mathbb{R}^{d}\right)$ be fixed so that $\left\|\phi_{i}\right\|_{L^{2}}=1$ for each $i$. Then, using the extended isometry property of the STFT (2), Hölder's inequality, and Proposition 1, for any $g \in \mathcal{M}^{p_{0}^{\prime}}, q_{0}^{\prime}$ we have

$$
\begin{aligned}
\left|\left\langle T_{\sigma} \vec{f}, g\right\rangle\right| & =\left|\left\langle\sigma, W_{m}(g, \vec{f})\right\rangle\right|=\left|\left\langle V_{W_{m}\left(\phi_{0}, \vec{\phi}\right)} \sigma, V_{W_{m}\left(\phi_{0}, \vec{\phi}\right)} W_{m}(g, \vec{f})\right\rangle\right| \\
& \leq\left\|V_{W_{m}\left(\phi_{0}, \vec{\phi}\right)} \sigma\right\|_{L^{p, 1}}\left\|V_{W_{m}\left(\phi_{0}, \vec{\phi}\right)} W_{m}(g, \vec{f})\right\|_{L^{p^{\prime}, \infty}} \\
& \leq C\|\sigma\|_{\mathcal{M}^{p, 1}} \prod_{i=1}^{m}\left\|f_{i}\right\|_{\mathcal{M}^{p_{i}, q_{i}}}\|g\|_{\mathcal{M}^{p_{0}^{\prime}, q_{0}^{\prime}}} .
\end{aligned}
$$

If $p_{0}^{\prime}, q_{0}^{\prime}<\infty$, then the duality properties of the modulation spaces imply that $T_{\sigma} \vec{f} \in \mathcal{M}^{p_{0}, q_{0}}$ with the norm estimate

$$
\left\|T_{\sigma} \vec{f}\right\|_{\mathcal{M}^{p_{0}, q_{0}}} \leq C\|\sigma\|_{\mathcal{M}^{\infty, 1}} \prod_{i=1}^{m}\left\|f_{i}\right\|_{\mathcal{M}^{p_{i}, q_{i}} .}
$$

If either $p_{0}^{\prime}=\infty$ or $q_{0}^{\prime}=\infty$ or both, then we take $g \in \mathcal{M}^{0, q_{0}^{\prime}}, \mathcal{M}^{p_{0}^{\prime}, 0}$, or $\mathcal{M}^{0,0}$ instead, and, following Remark 3 , a similar duality argument yields the desired result.

REMARK 5. If we let $p=1$ in Theorem 1 , we necessarily have $p_{0}=q_{0}=1$ and $p_{i}=q_{i}=\infty$ for $i=1, \ldots, m$. The theorem then reads as follows:

If $\sigma \in \mathcal{M}^{1}\left(\mathbb{R}^{(m+1) d}\right)$ then $T_{\sigma}$ is bounded from $\mathcal{M}^{\infty} \times \cdots \times \mathcal{M}^{\infty}$ into $\mathcal{M}^{1}$. 
Thus, since $\mathcal{M}^{1} \subset \mathcal{M}^{p, q} \subset \mathcal{M}^{\infty}$ for $1 \leq p, q \leq \infty$, we infer that symbols in the Feichtinger algebra produce bounded multilinear pseudodifferential operators from arbitrary products of modulation spaces into an arbitrary modulation space. This improves on our results in [4] where the technical tools we used required further restrictions on the indices. Furthermore, since $\mathcal{M}^{1} \subset L^{p} \subset \mathcal{M}^{\infty}$, it also follows from Theorem 1 that if $\sigma \in \mathcal{M}^{1}$, then $T_{\sigma}$ is bounded from any product of Lebesgue spaces into $\mathcal{M}^{1}$, thus into any $L^{p}$ space. These strong boundedness results are a consequence of the fact that symbols in $\mathcal{M}^{1}$, although non-smooth, have a considerable smoothing effect on the corresponding operators.

REMARK 6. If $p=\infty$, we recover the main result of [2]. In a sense, Theorem 1 should be viewed as a general result about a continuous family of spaces that yields bounded multilinear pseudodifferential operators on products of certain modulation spaces.

The next proposition provides us with the estimate needed in the proof of Theorem 2.

Proposition 2. Let $1 \leq p_{0}, p, q, r, q_{i} \leq \infty$ for $0 \leq i \leq m$ be such that

$$
\left\{\begin{array}{l}
p_{0} \leq q_{0} \\
q_{m} \leq r \\
p^{\prime} \leq q^{\prime}, q_{0}^{\prime}, r, q_{i} \quad \text { if } 1 \leq i \leq m, \\
q_{i} \leq q^{\prime} \quad \text { if } 1 \leq i \leq m-1
\end{array}\right.
$$

Moreover, assume that

$$
\frac{m-1}{p}+\frac{1}{q}+\sum_{i=1}^{m} \frac{1}{q_{i}}=m-1+\frac{1}{q_{0}} \quad \text { and } \quad \frac{1}{p}+\frac{1}{q}+\frac{1}{r}=1+\frac{1}{p_{0}} .
$$

Then

$$
\begin{aligned}
\left\|V_{W_{m}\left(\phi_{0}, \vec{\phi}\right)} W_{m}(g, \vec{f})\right\|_{L^{p^{\prime}, q^{\prime}}} & \\
& \leq C\left\|f_{1}\right\|_{\mathcal{M}^{q^{\prime}, q_{1}}} \cdots\left\|f_{m-1}\right\|_{\mathcal{M}^{q^{\prime}, q_{m-1}}}\left\|f_{m}\right\|_{\mathcal{M}^{r, q m}}\|g\|_{\mathcal{M}^{p_{0}^{\prime}, q_{0}^{\prime}}},
\end{aligned}
$$

whenever the right hand side is defined. Equivalently, $W_{m}(g, \vec{f}) \in \mathcal{M}^{p^{\prime}, q^{\prime}}$.

Proof. We recall from the proof of Proposition 1 that

$$
\begin{aligned}
& \Gamma\left(v_{0}, \vec{v}\right)^{p^{\prime}} \\
& \quad=\int_{\left(\mathbb{R}^{d}\right)^{m+1}}\left|V_{\phi_{0}} g\left(u_{0}, v_{0}+\sum_{i=1}^{n} u_{i}\right)\right|^{p^{\prime}}\left|\prod_{i=1}^{m} V_{\phi_{i}} f_{i}\left(u_{0}+v_{i}, u_{i}\right)\right|^{p^{\prime}} d u_{0} d \vec{u} .
\end{aligned}
$$

For a function $F(x, y)$ on $\mathbb{R}^{2 d}$, we let $\widetilde{F}(x, y)=F(x,-y)$. With this notation, (5) can be rewritten as an equality between $\Gamma$ and a convolution 
integral

$$
\begin{aligned}
\Gamma\left(v_{0}, \vec{v}\right)= & \left(\left.\int_{\mathbb{R}^{d}}\left|V_{\phi_{0}} g\left(u_{0}, \cdot\right)\right|^{p^{\prime}} * \widehat{\mid V_{\phi_{1}} f_{1}}\left(u_{0}+v_{1}, \cdot\right)\right|^{p^{\prime}} * \cdots\right. \\
& \left.*\left|\widehat{V_{\phi_{m}} f_{m}}\left(u_{0}+v_{m}, \cdot\right)\right|^{p^{\prime}}\left(v_{0}\right) d u_{0}\right)^{1 / p^{\prime}} .
\end{aligned}
$$

Here, the convolution is only in the second variable. Consequently,

$$
I=\int_{\mathbb{R}^{(m+1) d}} \Gamma\left(v_{0}, \vec{v}\right)^{q^{\prime}} d v_{0} d \vec{v}
$$

can be rewritten as

$$
\begin{aligned}
\int_{\mathbb{R}^{m d}} \int_{\mathbb{R}^{d}}\left(\int_{\mathbb{R}^{d}}\left|V_{\phi_{0}} g\left(u_{0}, \cdot\right)\right|^{p^{\prime}} *\right. & \left.\widehat{V_{\phi_{1}} f_{1}}\left(u_{0}+v_{1}, \cdot\right)\right|^{p^{\prime}} * \cdots \\
& \left.\left.* \widehat{\mid V_{\phi_{m}} f_{m}}\left(u_{0}+v_{m}, \cdot\right)\right|^{p^{\prime}}\left(v_{0}\right) d u_{0}\right)^{q^{\prime} / p^{\prime}} d v_{0} d \vec{v} .
\end{aligned}
$$

Since $q^{\prime} / p^{\prime} \geq 1$, Minkowski's inequality allows us to bound $I$ by $\int_{\mathbb{R}^{m d}} I I(\vec{v}) d \vec{v}$, where $I I(\vec{v})$ is given by the following expression:

$$
\begin{aligned}
\left(\int \left(\int \left(\left|V_{\phi_{0}} g\left(u_{0}, \cdot\right)\right|^{p^{\prime}}\right.\right.\right. & \left.* \widehat{\mid V_{\phi_{1}} f_{1}}\left(u_{0}+v_{1}, \cdot\right)\right|^{p^{\prime}} * \ldots \\
& \left.\left.\left.*\left|\widehat{V_{\phi_{m}} f_{m}}\left(u_{0}+v_{m}, \cdot\right)\right|^{p^{\prime}}\left(v_{0}\right)\right)^{q^{\prime} / p^{\prime}} d v_{0}\right)^{p^{\prime} / q^{\prime}} d u_{0}\right)^{q^{\prime} / p^{\prime}} .
\end{aligned}
$$

Using now the conditions on the indices and Young's inequality we can further estimate $\int_{\mathbb{R}^{m d}} I I(\vec{v}) d \vec{v}$, and get

$$
I \leq \int_{\mathbb{R}^{m d}}\left(\int_{\mathbb{R}^{d}}\left\|V_{\phi_{0}} g\left(u_{0}, \cdot\right)\right\|_{L^{q_{0}^{\prime}}}^{p^{\prime}} \prod_{i=1}^{m}\left\|V_{\phi_{i}} f_{i}\left(u_{0}+v_{i}, \cdot\right)\right\|_{L^{q_{i}}}^{p^{\prime}} d u_{0}\right)^{q^{\prime} / p^{\prime}} d \vec{v} .
$$

By a repeated use of Minkowski's inequality to each of the variables $v_{i}$, $i=1, \ldots, m$, we obtain

$$
\begin{aligned}
I \leq & \prod_{i=1}^{m-1} \int_{\mathbb{R}^{d}}\left\|V_{\phi_{i}} f_{i}\left(v_{i}, \cdot\right)\right\|_{L^{q_{i}}}^{q^{\prime}} \\
& \times \int_{\mathbb{R}^{d}}\left(\left\|V_{\phi_{0}} g\left(u_{0}, \cdot\right)\right\|_{L^{q_{0}^{\prime}}}^{p^{\prime}}\left\|V_{\phi_{m}} f_{m}\left(u_{0}+v_{m}, \cdot\right)\right\|_{L^{q_{m}}}^{p^{\prime}} d u_{0}\right)^{q^{\prime} / p^{\prime}} d \vec{v} \\
= & \prod_{i=1}^{m-1} \int_{\mathbb{R}^{d}}\left\|V_{\phi_{i}} f_{i}\left(v_{i}, \cdot\right)\right\|_{L^{q_{i}}}^{q^{\prime}} \int_{\mathbb{R}^{d}}\left(G_{0} * F_{m}\left(v_{m}\right)\right)^{q^{\prime} / p^{\prime}} d \vec{v}
\end{aligned}
$$

where we have set

$$
G_{0}\left(u_{0}\right)=\left\|V_{\phi_{0}} g\left(u_{0}, \cdot\right)\right\|_{L^{q_{0}^{\prime}}}^{p^{\prime}} \quad \text { and } \quad F_{m}\left(u_{0}\right)=\left\|V_{\phi_{m}} f_{m}\left(u_{0}, \cdot\right)\right\|_{L^{q_{m}}}^{p^{\prime}} .
$$


Now, it is easy to see that for $i=1, \ldots, m-1$, we have on the one hand,

$$
\int_{\mathbb{R}^{d}}\left\|V_{\phi_{i}} f_{i}\left(v_{i}, \cdot\right)\right\|_{L^{q_{i}}}^{q^{\prime}} d v_{i} \leq\left\|f_{i}\right\|_{\mathcal{M}^{q^{\prime}, q_{i}}}^{q^{\prime}},
$$

while on the other hand,

$$
\int_{\mathbb{R}^{d}}\left(G_{0} * F_{m}\left(v_{m}\right)\right)^{q^{\prime} / p^{\prime}} d v_{m} \leq\|g\|_{\mathcal{M}^{p_{0}^{\prime}, q_{0}^{\prime}}}^{q^{\prime}}\left\|f_{m}\right\|_{\mathcal{M}^{r, q_{m}}}^{q^{\prime}} .
$$

Consequently, from (6) and (7), we conclude that

$$
\begin{aligned}
\left\|W_{m}(g, \vec{f})\right\|_{\mathcal{M}^{p^{\prime}, q^{\prime}}} & =\left(\int_{\mathbb{R}^{(m+1) d}} \Gamma\left(v_{0}, \vec{v}\right)^{q^{\prime}} d v_{0} d \vec{v}\right)^{1 / q^{\prime}} \\
& \leq C\|g\|_{\mathcal{M}^{p_{0}^{\prime}, q_{0}^{\prime}}} \prod_{i=1}^{m-1}\left\|f_{i}\right\|_{\mathcal{M}^{q^{\prime}, q_{i}}}\left\|f_{m}\right\|_{\mathcal{M}^{r, q_{m}}},
\end{aligned}
$$

where $C$ is a positive constant depending only on the dimension and the indices of the spaces involved.

Proof of Theorem 2. The proof, making use of Proposition 2, is modeled after the proof of Theorem 1 with the obvious changes. The details are left to the interested reader.

If we choose $q=p^{\prime}$ in Theorem 2 , then $p_{0}=r$ and we obtain a restricted generalization of symbols in $\mathcal{M}^{\infty, 1}$ to symbols in $\mathcal{M}^{p, p^{\prime}}$; see Remark 6 .

Corollary 1. Let $p \geq 2$ and $1 \leq r, q_{i} \leq \infty$ for $0 \leq i \leq m$ be such that

$$
\left\{\begin{array}{l}
r \leq q_{0}, \\
q_{m} \leq r \\
p^{\prime} \leq q_{0}^{\prime}, r, q_{i} \quad \text { if } 1 \leq i \leq m, \\
q_{i} \leq q^{\prime} \text { if } 1 \leq i \leq m-1
\end{array}\right.
$$

Moreover, assume that

$$
\frac{m-2}{p}+\sum_{i=1}^{m} \frac{1}{q_{i}}=m-2+\frac{1}{q_{0}} .
$$

If $\sigma \in \mathcal{M}^{p, p^{\prime}}\left(\mathbb{R}^{(m+1) d}\right)$ then $T_{\sigma}$ can be extended to a bounded operator from $\mathcal{M}^{p, q_{1}} \times \cdots \times \mathcal{M}^{p, q_{m-1}} \times \mathcal{M}^{r, q_{m}}$ into $\mathcal{M}^{r, q_{0}}$.

With some modifications in the proof of Proposition 2, we can also obtain the following result.

Corollary 2. Let $1 \leq p, q, q_{i} \leq \infty$ for $0 \leq i \leq m$ be such that $q \leq p$ $\leq q_{0}$ and $p^{\prime} \leq q_{i} \leq q^{\prime}$ for all $1 \leq i \leq m$. Moreover, assume that

$$
\frac{m-1}{p}+\frac{1}{q}+\sum_{i=1}^{m} \frac{1}{q_{i}}=m-1+\frac{1}{q_{0}} .
$$


If $\sigma \in \mathcal{M}^{p, q}\left(\mathbb{R}^{(m+1) d}\right)$, then $T_{\sigma}$ can be extended to a bounded operator from $\mathcal{M}^{q^{\prime}, q_{1}} \times \cdots \times \mathcal{M}^{q^{\prime}, q_{m}}$ into $\mathcal{M}^{p, q_{0}}$.

REMARK 7 . The condition $p \geq q$ appears naturally in the hypothesis of Theorem 2 or Corollary 2 due to the extensive use of Minkowski's inequality. Furthermore, the absence of this condition has rather unexpected consequences. If we assume, for example, that $1 \leq p \leq 2$ and $\sigma \in \mathcal{M}^{1, p}\left(\mathbb{R}^{(m+1) d}\right)$, then it can be shown that $T_{\sigma}$ is bounded from $L^{p} \times \cdots \times L^{p}$ into $L^{p}$. However, if $p \geq 2$ and $\sigma \in \mathcal{M}^{1, p}\left(\mathbb{R}^{(m+1) d}\right)$, then $T_{\sigma}$ might actually fail to be bounded on products of Lebesgue spaces. Indeed, if we let $\sigma=\delta_{0}$, the Dirac (point mass) distribution at the origin, then it is easy to see that $\sigma \in \mathcal{M}^{1, \infty}\left(\mathbb{R}^{(m+1) d}\right)$, but $T_{\sigma}$ is not bounded on any product of Lebesgue spaces. It is not clear to these authors how to proceed in the case $p<q<\infty$.

\section{References}

[1] Á. Bényi, Bilinear pseudodifferential operators with forbidden symbols on Lipschitz and Besov spaces, J. Math. Anal. Appl. 284 (2003), 97-103.

[2] Á. Bényi, K. Gröchenig, C. Heil, and K. Okoudjou, Modulation spaces and a class of bounded multilinear pseudodifferential operators, J. Operator Theory 54 (2005), 301-313.

[3] Á. Bényi, A. R. Nahmod, and R. H. Torres, Sobolev space estimates and symbolic calculus for bilinear pseudodifferential operators, preprint.

[4] Á. Bényi and K. Okoudjou, Bilinear pseudodifferential operators on modulation spaces, J. Fourier Anal. Appl. 10 (2004), 301-313.

[5] Á. Bényi and R. H. Torres, Almost orthogonality and a class of bounded bilinear pseudodifferential operators, Math. Res. Lett. 11 (2004), 1-11.

[6] - - - Symbolic calculus and the transposes of bilinear pseudodifferential operators, Comm. Partial Differential Equations 28 (2003), 1161-1181.

[7] Á. Bényi and N. Tzirakis, Multilinear almost diagonal estimates and applications, Studia Math. 164 (2004), 75-89.

[8] A. P. Calderón and R. Vaillancourt, On the boundedness of pseudodifferential operators, J. Math. Soc. Japan 23 (1971), 374-378.

[9] R. R. Coifman and Y. Meyer, On commutators of singular integrals and bilinear singular integrals, Trans. Amer. Math. Soc. 212 (1975), 315-331.

[10] —, - Au-delà des opérateurs pseudo-différentiels, Astérisque 57 (1978).

[11] - - - Commutateurs d'intégrales singulières et opérateurs multilinéaires, Ann. Inst. Fourier (Grenoble) 28 (1978), no. 3, 177-202.

[12] W. Czaja, Boundedness of pseudodifferential operators on modulation spaces, J. Math. Anal. Appl. 284 (2003), 389-396.

[13] H. G. Feichtinger, On a new Segal algebra, Monatsh. Math. 92 (1981), 269-289.

[14] G. B. Folland, Harmonic Analysis in Phase Space, Ann. of Math. Stud. 122, Princeton Univ. Press, Princeton NJ, 1989.

[15] K. Gröchenig, Foundations of Time-Frequency Analysis, Birkhäuser, Boston, 2001.

[16] K. Gröchenig and C. Heil, Modulation spaces and pseudodifferential operators, Integral Equations Oper. Theory 34 (1999), 439-457. 
[17] K. Gröchenig and C. Heil, Modulation spaces as symbol classes for pseudodifferential operators, in: Proc. ICWA, Chennai, 2002, 151-170.

[18] - - - Counterexamples for boundedness of pseudodifferential operators, Osaka J. Math. 41 (2004), 681-691.

[19] L. Grafakos and R. H. Torres, A multilinear Schur test and multiplier operators, J. Funct. Anal. 187 (2001), 1-24.

[20] —, - Discrete decompositions for bilinear operators and almost diagonal estimates, Trans. Amer. Math. Soc. 354 (2002), 1153-1176.

[21] C. Heil, J. Ramanathan and P. Topiwala, Singular values of compact pseudodifferential operators, J. Funct. Anal. 150 (1997), 426-452.

[22] D. Labate, Pseudodifferential operators on modulation spaces, J. Math. Anal. Appl. 262 (2001), 242-255.

[23] - , Time-frequency analysis of pseudodifferential operators, Monatsh. Math. 133 (2001), 143-156.

[24] K. Okoudjou, Embeddings of some classical Banach spaces into modulation spaces, Proc. Amer. Math. Soc. 132 (2004), 1639-1647.

[25] J. Sjöstrand, An algebra of pseudodifferential operators, Math. Res. Lett. 1 (1994), $185-192$.

[26] J. Toft, Continuity properties for modulation spaces, with applications to pseudodifferential operators. I, J. Funct. Anal. 207 (2004), 399-429.

Department of Mathematics

Western Washington University

516 High Street

Bellingham, WA 98225-9063, U.S.A.

E-mail: arpad.benyi@wwu.edu
Department of Mathematics Cornell University Malott Hall

Ithaca, NY 14853-4201, U.S.A. E-mail: kasso@math.cornell.edu

Received December 7, 2004

Revised version September 20, 2005 